\section{Lower extremity swelling in a patient with psoriatic arthritis}

\author{
Rebekah Condit, Guy Fiocco \\ Scott and White Healthcare, Texas A\&M \\ Health Science Center College of \\ Medicine, Temple, TX, USA
}

\begin{abstract}
Swelling of an extremity secondary to psoriatic arthritis is a rare condition that typically occurs unilaterally, in the upper extremities, but can progress to involve multiple extremities. We report a patient with psoriatic arthritis who developed lower extremity swelling consistent with lymphedema that was unresponsive to traditional therapy. The pathophysiology of this disease may involve either tenosynovial involvement or lymphatic dysfunction. Clinical exam may help differentiate between the two types, and magnetic resonance imaging and/or lymphoscintigraphy can be used for more definitive diagnosis. In addition, other causes of extremity edema need to be evaluated. Recommendations for diagnosis and treatment of patients with this disease are discussed.
\end{abstract}

\section{Introduction}

Swelling of an extremity secondary to psoriatic arthritis is a rare condition, with twelve cases reported in the literature. The condition typically occurs unilaterally, in the upper extremities, but can progress to involve multiple extremities. Previous case reports have discussed the characteristics and the pathophysiology of the disease and the efficacy of various treatments, including TNF- $\alpha$ antagonists and corticosteroids.

We are reporting a case of a patient with psoriatic arthritis who developed lower extremity swelling consistent with lymphedema that was unresponsive to traditional therapy. We conclude with general clinical recommendations for diagnosis and treatment of patients with this disease.

\section{Case Report}

A 44-year-old Caucasian female presented with a 1.5 year history of psoriasis involving her scalp, legs and buttock. The patient had been previously treated by a dermatologist with floucinonide (Lidex) solution, floucinonide (Lidex) ointment and tacrolimus ointment
(Protopic) with minimal resolution of her psoriasis. The patient developed joint symptoms, intermittent swelling of her knees, discomfort without swelling in her hands, and 1-2 hours of morning stiffness. She began taking $800 \mathrm{mg}$ Ibuprofen 2-3 pills a day with some improvement of symptoms. Methotrexate, (MTX) (15 mg per week) was added to the regimen for presumed psoriatic arthritis (PsA). After a month of MTX use, an elevation in liver transaminases led to discontinuation. Her dermatologist started her on Enbrel (etanercept $50 \mathrm{mg}$ ) and referred her to the Rheumatology clinic.

The patient had been taking etanercept for one month, with alleviation of her skin lesions, but the patient reported no improvement of joint symptoms. She continued to have 1-2 hours of morning stiffness and persistent discomfort in hands and wrists with intermittent low back pain. For the past year, the swelling of her right ankle and foot had been severe enough to prevent her from wearing shoes on that foot.

Her past medical history included hypertension, mitral valve prolapse and pneumonia on 2 occasions. Social history is notable for her employment in a clerical position; she is single and quit smoking over 10 years ago. Family history is remarkable for a mother who had severe rheumatoid arthritis. She has two children, both of whom are healthy. Review of systems is otherwise unremarkable.

On physical exam, the patient was afebrile, hypertensive and moderately obese. There were minimal skin findings on exam. Her extremities showed no clubbing or cyanosis. There was firm, slightly pitting edema in the right ankle and mild edema in the left ankle. The edema extended up the right leg threefourths of the way to the knee. The knees showed good range of motion, minimal tenderness and no effusions. There was tenderness to palpation over the malleoli bilaterally, tenderness with inversion and eversion of the ankle, and slight warmth to palpation on the right side compared to the left. There was minimal tenderness to compression of her metacarpophalangeal (MTPs) (Figure 1).

The rest of the exam was normal, with no hepatosplenomegaly, adenopathy, or thyromegaly. Her antinuclear antibody (ANA) profile, rheumatoid factor, and hepatitis panel were negative, and HLA-B27 was not present. Other labs included CCP-IGG $<20$, CRP (Creactive protein) below assay limit, ESR (Erythrocyte sedimentation rate) 28 (0-20), TSH (thyroid stimulating hormone) 0.93 (0.44). Imaging studies included hand $\mathrm{X}$-rays that showed bilateral joint space narrowing of the $1^{\text {st }}$ IP joint and the $5^{\text {th }}$ MCP joint. The right radiocarpal joint was narrowed. The left hand showed narrowing of the radiocarpal joint, the $5^{\text {th }}$ MCP joint with ulnar subluxation.
Correspondence: Guy Fiocco, Texas A\&M Health Science Center College of Medicine, 2401 South 31st Street, Temple, TX 76508, USA.

Tel. +1.254.743.2285 - Fax: +1 254.743.0121

E-mail: guy.fiocco@va.gov

Key words: psoraiatic arthritis; lower extremity swelling; lymphedema; DMARD therapy.

Contributions: the authors contributed equally.

Conflict of interest: the authors declare no potential conflict of interests.

Received for publication: 8 May 2012.

Accepted for publication: 17 September 2012

This work is licensed under a Creative Commons Attribution NonCommercial 3.0 License (CC BYNC 3.0).

(C) Copyright R. Condit and G. Fiocco, 2013

Licensee PAGEPress, Italy

Rheumatology Reports 2013; 5:el

doi:10.4081/rr.2013.el

A duplex ultrasound was completed on the right leg and pelvis which ruled out a deep venous thrombosis (DVT), and a trial of support hose, metolazone and furosemide was completed with minimal improvement.

\section{Clinical course}

A trial of prednisone $10 \mathrm{mg}$ twice daily initially resolved the swelling, but after a decrease in the dose to $5 \mathrm{mg}$ twice daily, the edema recurred. Etanercept was increased to twice weekly, and methotrexate was added back at $15.0 \mathrm{mg}$ per week. In contrast to her previous use of methotexate, there was no elevation of transaminases. However, no clinical improvement was seen. Four months after beginning etanercept, this medication was switched to adulimumab $40 \mathrm{mg}$ every two weeks with no improvement in her condition. Seven months later the patient developed superimposed erythema of the right leg, diagnosed as cellulitis, and the swelling of the right leg appeared to be worse than the initial presentation. Re-evaluation for DVT was undertaken. A computed tomography (CT) of the abdomen/pelvis was negative, but a duplex ultrasound demonstrated a thrombus located from mid-calf to mid-thigh in the right leg. A hypercoaguable workup was negative and an anti-coagulation was initiated. Adulimumab and methotrexate were held due to superimposed-infection. Physical therapy for edema was started. Once the infection cleared, methotrexate was restarted with minimal improvement noted. Our patient showed par- 
tial response to intermittent trials of steroids but there was no complete resolution of the leg swelling. The presumptive diagnosis of lymphedema was made and further physical therapy was initiated utilizing mechanical decompression affording her additional relief of the swelling.

\section{Discussion}

\section{Extremity swelling associated with psoriatic arthritis}

Lymphedema is defined as an accumulation of tissue fluid secondary to impaired lymphatic drainage (Szuba). There have been twelve cases of distal extremity swelling secondary to PsA reported in the literature. The diagnosis of lymphedema is usually clinical, although it can be diagnosed using isotopic lymphoscintigraphy, indirect and direct lymphography, magnetic resonance imaging (MRI), CT, and ultrasound if the history and physical exam do not make the diagnosis clear. ${ }^{1}$ The two most commonly used methods in the literature are lymphoscintigraphy and MRI. Direct lymphography is rarely used clinically except for those patients who require lymphatic surgery. ${ }^{1}$

There have been fewer than 30 case reports of lymphedema associated with rheumatoid arthritis in the literature, ${ }^{2}$ and this condition has been rarely recognized in PsA. In rheumatoid arthritis the edema has been reported in both the upper and lower extremities and is felt to be a complication to the synovitis. There may also be increased capillary permeability or abnormal lymphatic function leading to swelling and edema. ${ }^{3}$

Swelling in PsA is more likely to occur in the upper extremities. The onset of edema is unpredictable and is not related to arthritis duration or severity, although radiologic joint damage progression is related to edema progression. ${ }^{4}$ There has been some evidence by Kiely et al. that showed that inflammatory arthritis by itself does not cause impaired lymphatc flow, suggesting that the lymphedema secondary to PsA is multi-factorial. ${ }^{5}$ Quarta et al. discussed the different pathologic mechanisms that produce swelling in the setting of PsA. Using lymphoscintigraphy and MRI, the researchers differentiated two distinct causes of extremity distention: i) tenosynovial inflammation and ii) lymphatic vessel dysfunction. ${ }^{6}$ The authors suggested that patients with tenosynovial involvement without lymphatic changes had a more favorable outcome to teatment. In our patient, who showed no response to standard treatment options, lymphatic involvement may explain her poor outcome to therapy.

In a case-control study by Cantini et al., the frequency of distal extremity swelling related to PsA was evaluated. Despite only twelve cases in the literature diagnosed clinically as lymphedema secondary to PsA, their research showed that this side effect of PsA may be more common than originally thought. In their study 183 patients with PsA were compared to 366 outpatient rheumatic disease patients with other diagnosis. They found that $21 \%$ of PsA patients had edema versus $4.9 \%$ of controls, and in $20 \%$ of patients distal extremity edema was the first clinical manifestation of PsA. ${ }^{7}$ The researchers classified patients through physical exam into those suspected of having swelling related to tenosynovial inflammation versus those whose swelling related to lymphatic involvement. Clinically, tenosynovitis was diagnosed by pain and swelling over tenosynovial structures and reproducible pitting, whereas swelling due to lymphatic involvement was less painful and more evenly distributed. In the seven patients suspected of having tenosynovial-related lymphedema, a MRI was completed which showed marked soft tissue edema as well as tenosynovial involvement. Lymphatic involvement in patients considered to have tenosynovitis, could not be excluded on clinical grounds alone without MRI or lymphoscintigraphy. Cantini found that edema secondary to suspected lymphatic damage had a poor response to therapy, whereas tenosynovitis-related edema rapidly responded to corticosteroid therapy.
This report was largely based on retrospective chart review and patient self reporting. Only seven patients had an MRI to confirm the tenosynovitis. Most of these patients had only one episode that promptly resolved after 1-2 months of $12.5 \mathrm{mg}$ of prednisolone. This research suggests that distal extremity swelling with inflammatory arthritis, specifically psoriatic arthritis, may be more common than currently recognized and appropriate treatment depends on the pathophysiologic cause of distal extremiy swelling.

\section{Recommendations for evaluation}

The diagnosis of extremity swelling in PsA can be more complex than a simple clinical diagnosis. Cantini et al. suggest that pain and pitting edema located along a specific tendon may be related to tenosynovitis, whereas swelling due to lymphatic dysfunction may be less painful and more widespread. ${ }^{7}$ As in our patient, intervening venous thrombosis may complicate the picture and needs to remain in the differential diagnosis; therefore, venous duplex ultrasonography should be performed if this diagnosis is suspected. MRI and lymphoscintigraphy are options to help clarify the diagnosis but are expensive and invasive. Other complications include skin breakdown, psychological issues associated with physical appearance, decreased mobility and function leading to decreased quality of life.
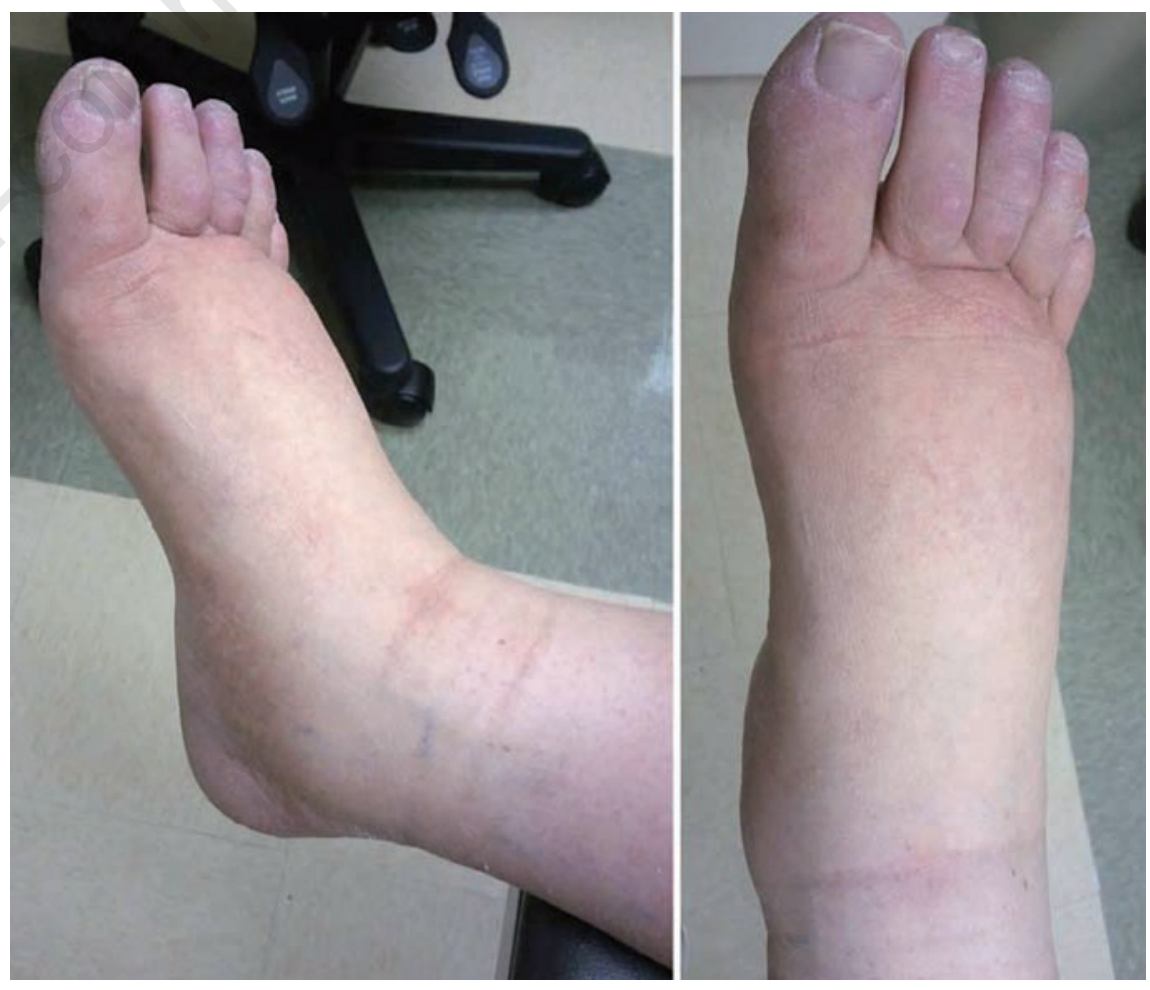

Figure 1. Diffuse lower extremity edema in psoriatic arthritis. 


\section{Treatment}

There are several treatment options for lymphedema related to psoriatic arthritis. Initial treatment may be a trial of corticosteroids to judge responsiveness. If there is no response to steroids, physical therapy with mechanical compression should be considered. If response to steroids is favorable, medical therapy may then include disease-modifying anti-rheumatic drugs (DMARDs), and anti-TNF biologics. If medical and physical therapy fail, then microsurgery, debulking procedures, and autologous lymphocyte injections may be explored. As discussed above, our patient did not have complete resolution with multiple therapies including the TNF antagonists. Further research is needed to determine the exact cause of lymphedema in order to adequately target treatment options.

In addition to a small study, Salvarani et al. suggested that the response to steroids may depend on the pathophysiologic cause of distal extremity swelling. Two cases were discussed, one caused by tenosynovial inflammation and the other by lymphatic dysfunction (confirmed by MRI and lymphoscintigraphy respectively), which showed the effectiveness of corticosteroids. ${ }^{8}$ In this case report, steroids were successful in resolving edema secondary to tenosynovitis but had no effect on treating primary lymphedema. As discussed earlier, the Cantini study demonstrated that corticosteroids may have a positive result in treating swelling secondary to PsA in patients with predominately tenosynovial involvement, but may be ineffective for patients with lymphatic dysfunction. ${ }^{7}$ Our patient had a partial response to steroids without complete resolution, and without an MRI or lymphoscintigraphy to determine the exact cause of swelling; we are left to assume it was more lymphatic-related based on the lack of response to steroids.

In a case report by Quarta et al. the efficacy of etanercept, a TNF- $\alpha$ antagonist, was investigated. Two patients with MRI- and/or lymphoscintigraphy-confirmed tenosynovitis or lymphedema were placed on a trial of etanercept. Both patients' arthritis symptoms benefit- ed from treatment, but there was no improvement in lymphedema. ${ }^{6}$ Lepka et al. reported a patient with PsA and related left upper arm swelling who was successfully treated with etanercept therapy. ${ }^{9}$ Our patient's lymphedema did not improve significantly after DMARD therapy. These cases support a trial of TNF- $\alpha$ antagonists anticipating improvement in arthritic symptoms and possibly PsA related swelling.

Several case reports include exercise programs as an important part of therapy. Exercise has been used as monotherapy or in combination with compression hosiery, heat therapy, and/or medical therapies. Mulherin reported a case where edema persisted despite years of therapy when an exercise program was used with compression hosery alone with no medications. ${ }^{4}$ Complex decongestive physiotherapy (CDP) involves skin care education, manual lymphatic drainage, multi-layer compression bandages, and home exercises. ${ }^{10}$ When used in psoriasis-related lymphedema, CDP has shown success in reducing patient discomfort and improving function and range of motion. Physical therapy should be used in conjunction with medical therapy in the treatment of lymphedema secondary to psoriasis.

\section{Conclusions}

We report a rare case of lymphedema of the lower extremity associated with psoriatic arthritis. The pathophysiology of this disease may involve either tenosynovial involvement or lymphatic dysfunction. Clinical exam may help differentiate between the two types, and MRI and/or lymphoscintigraphy can be used for more definitive diagnosis. However, these studies may not necessarily determine the optimal therapeutic approach. We recommend treating patients initially with corticosteroids. If there is a response to steroids, the patient may have tenosynovial involvement and may benefit from more extensive therapy including a change in DMARDs or the addition of anti-TNF therapy. If there is no response to steroids the patient may have lymphatic dysfunction causing the edema, and further treatment may center on physical therapy and symptomatic treatment.

\section{References}

1. Szuba A, Rockson S. Lymphedema: classification, diagnosis, and therapy. Vasc Med 1998;3:145-56.

2. Ostrov BE. Beneficial effect of etanercept on rheumatoid lymphedema. Arthritis Rheum 2001;44:240-1.

3. Almodovar R, Zarco P, Quiros FJ, Mazzucchelli R. Infliximab treatment efficacy in lymphoedema associated with ankylosing spondylitis. Rheumatology 2004;43:1456.

4. Mulherin DM, FitzGerald 0, Bresnihan B. Lymphedema of the upper limb in patients with psoriatic arthritis. Semin Arthritis Rheum 1993;22:350-6.

5. Kiely PW, Band JM, Joseph AE, et al. Upper limb lymphatic function in inflammatory arthritis. J Rheumatol 1995;22:214-7.

6. Quarta L, Corrado A, d'Onofrio F, et al. Two cases of distal extremity swelling with pitting oedema in psoriatic arthritis: the different pathological mechanisms. Rheumatol Int 2010;30:1367-70.

7. Cantini F, Salvarani C, Olivieri I, et al. Distal extremity swelling with pitting edema in psoriatic arthrits: a case-control study. Clin Exp Rheumatol 2001;19:291-6.

8. Salvarani C, Cantini F, Olivieri I, et al. Distal extremity swelling with pitting edema in psoriatic arthritis: evidence of 2 pathological mechanisms. J Rheumatol 1999;26:1831-4.

9. Lekpa FK, Economu-Dubosc A, Fevre C, et al. Efficacy of etanercept in lymphedema associated with psoriatic arthritis. J Rheumatol 2009;36:207-8.

10. Kaya S, Akbayrak T, Bakar Y, Topuz S. Effects of complex decongestive physiotherapy on an older female patient with psoriasis-related primary lymphedema. Top Geriatr Rehabil 2010;26:171-5. 